\title{
DNA Multiple Sequence Alignment by a Hidden Markov Model and Fuzzy Levenshtein Distance based Genetic Algorithm
}

\author{
Tamal Chakrabarti \\ Department of Computer \\ science \& Engineering, Institute \\ of Engineering and \\ Management, Y-12, Block -EP, \\ Sector-V, Salt Lake Electronics \\ Complex, Kolkata-700091, \\ West Bengal, India
}

\author{
Sourav Saha \\ Department of Computer \\ science \& Engineering, Institute \\ of Engineering and \\ Management, Y-12, Block -EP, \\ Sector-V, Salt Lake Electronics \\ Complex, Kolkata-700091, \\ West Bengal, India
}

\author{
Devadatta Sinha \\ Department of Computer \\ Science \& Engineering, \\ Calcutta University, 92 \\ AcharyaPrafulla Chandra Road, \\ Kolkata-700009, West Bengal, \\ India
}

\begin{abstract}
In the last decade, biologists have experienced a fundamental shift away from the traditional empirical research to largescale, computer-based research. Today bio-informatics is a systematic and predictive discipline which encompasses genomics, informatics, automation, and miniaturization. This fusion of biology and information science is expected to continue and expand for the foreseeable future. DNA Sequence alignment is a commonly observed problem in bioinformatics for establishing similarity and evolutionary relationship between DNA sequences. This paper has presented a DNA multiple sequence alignment technique by a genetic algorithm based on Hidden Markov Model and Fuzzy Levenshtein Distance.
\end{abstract}

\section{General Terms}

Bio-informatics, DNA, Sequence Alignment

\section{Keywords}

Genetic Algorithm, Hidden Markov Model, Fuzzy Levenshtein Distance

\section{INTRODUCTION}

DNA is short form for deoxyribonucleic acid. Two chains of four chemical bases (abbreviated A, T, C and G) make up DNA and act as a cell's recipe book to make proteins. The four bases in DNA are adenine $(A)$, thymine $(T)$, guanine $(G)$, and cytosine (C). DNA contains three components: deoxyribose (a five-carbon sugar), a series of phosphate groups, and four nitrogenous bases, (nitrogen compounds that contain bases). Adenine (A) forms a base pair with thymine $(\mathrm{T})$, and guanine $(\mathrm{G})$ with cytosine $(\mathrm{C})$ in DNA.

Similarities between DNA sequences may arise due to the functional, structural or evolutionary relationship among them. DNA Sequence alignment is a method of arranging two (pairwise) or more (multiple) sequences of DNA to identify regions of similarity among them by searching for a series of individual nucleotides or nucleotide patterns that are in the same order. In bioinformatics multiple sequence alignment is important in the study of evolution, control of gene expression and also in protein structure/function relationships. The figure below depicts an example of an alignment of three DNA sequences.

\begin{tabular}{|ccccc|}
\hline C & A & G & A & T \\
- & A & G & A & - \\
C & A & - & A & T \\
\hline
\end{tabular}

Figure 1: An example of Multiple DNA Sequence Alignment

The gap characters '-' are inserted in sequences to enforce regions of similarity to come together and form a good alignment. The quality of the alignment needs to be measured using some heuristic. The most commonly used method is sum-of-pairs score. Let $\mathrm{S}_{\mathrm{i}, \mathrm{j}}$ be the sum-of-pairs score of the alignment between $a_{1} \ldots a_{i}$ and $b_{1} \ldots b_{j}$ where $A=a_{1} \ldots a_{m}$ and $\mathrm{B}=\mathrm{b}_{1} \ldots \mathrm{b}_{\mathrm{n}}$ is the first and second sequence respectively. Then the recurrence relation to calculate $S_{i, j}$ is given by:

$$
S_{i, j}=\max \left(\begin{array}{c}
S_{i-1, j-1}+\varphi\left(a_{i}, b_{j}\right), \\
S_{i-1, j}+\varphi\left(a_{i},-\right), \\
S_{i, j-1}+\varphi\left(-, b_{j}\right)
\end{array}\right)
$$

Where:

$\varphi(\mathrm{a}, \mathrm{b})$ : scores an alignment of symbol a with symbol b. $\varphi(\mathrm{a},-)$ : scores an alignment of symbol a with a gap. $\varphi(-, b)$ : scores an alignment of a gap with symbol b.

The problem of finding the best possible alignment for multiple sequences simultaneously is NP-Hard. Therefore instead of finding the best alignment there has been extensive research done to find a near optimal solution using soft computing techniques such as Genetic Algorithm (GA). Genetic algorithm is an adaptive search heuristic in the field of Artificial Intelligence that imitates the process of natural evolution. It works on a philosophy of "natural selection" and genetic operators like crossover and mutation to pick the best among the candidates from a generation. For calculating the fitness of a candidate Hidden Markov Models (HMM) have been used. 
HMMs offer a way to model the latent structure of temporally dependent data where it is assumed that the observed process evolves independently given an unobserved Markov chain. There are a discrete finite number of states in the Markov chain which switch between one another according to a small probability. Given that these states are unobserved and random in occurrence they form a hidden Markov chain. It is possible to model the sequence of state changes that occur in the hidden Markov chain via observations which are dependent on the hidden states.

\section{RELATED WORK}

Hidden Markov models were first discussed by Baum and Petrie (1966) ${ }^{[1]}$. Since the 1980's and early 1990's HMMs have been applied to DNA sequence analysis with the seminal paper by Churchill (1989) that first applied HMM to DNA segmentation ${ }^{[4]}$. There are various statistical techniques available to assist in the segmentation effort which is covered in Braun and Muller (1998) ${ }^{[2]}$. The use of hidden Markov models in DNA sequence analysis were illustrated by Churchill (1992) ${ }^{[5]}$ and Dubin et al. (1998) ${ }^{[7]}$. Eddy et. al. (1995) ${ }^{[8]}$ presented a paper on DNA multiple sequence alignment by Hidden Markov Model. Genetic Algorithm has been applied to the multiple sequence alignment problem by various researchers, such as Lin et. al. ${ }^{[13]}$ and Chen et. al. ${ }^{[19]}$. Zhang et. al. have proposed ${ }^{[9]}$ a novel method of population initialization and of crossover. Chang et. al. [17] has successfully combined fuzzy arithmetic with GA to arrive at better alignments. Lai et. al. ${ }^{[3]}$ have suggested new genetic operators that direct the GA towards better solutions. Nguyen et al. ${ }^{[12]}$ presents a hybrid scheme where they convert the MSA problem to the problem of finding the shortest path in a weighted directed acyclic k-dimension graph (where $\mathrm{k}$ is the number of sequences). Hjelmqvist ${ }^{[10]}$ in 2012 published the idea of a fast and memory efficient Levenshtein algorithm to compute the edit distance between strings, such as DNA sequences. This paper presents a genetic algorithm, based on Hidden Markov Model and Fuzzy Levenshtein Distance to align multiple DNA sequences.

\section{ALGORITHM}

Given n DNA sequences, the genetic algorithm creates an initial population of chromosomes, by randomly generating $\mathrm{m}$ alignments of the given DNA sequences. For example, let CAGAT, AGA and CAAT be three given DNA sequences. Then an initial population of three randomly created chromosomes may be as depicted in the figure below:

\begin{tabular}{|ccccc|ccccc|ccccc|}
\hline C & A & G & A & T & C & A & G & A & T & C & A & G & A & T \\
A & - & G & - & A & A & G & - & A & - & A & - & G & - & A \\
C & A & A & T & - & C & A & - & A & T & C & A & - & A & T \\
\hline
\end{tabular}

Figure 2: Initial Population of Chromosomes

Then the fitness of the chromosomes is calculated by a fitness function.

\subsection{Fitness function}

For the purpose of calculating the fitness of each chromosome Hidden Markov Models (HMMs) are used. For every chromosome its profile HMM is built. A profile HMM is a certain type of HMM with a structure that in a natural way allows position dependent gap penalties. A profile HMM can be obtained from a multiple alignment. The structure of the model is shown in the figure below.

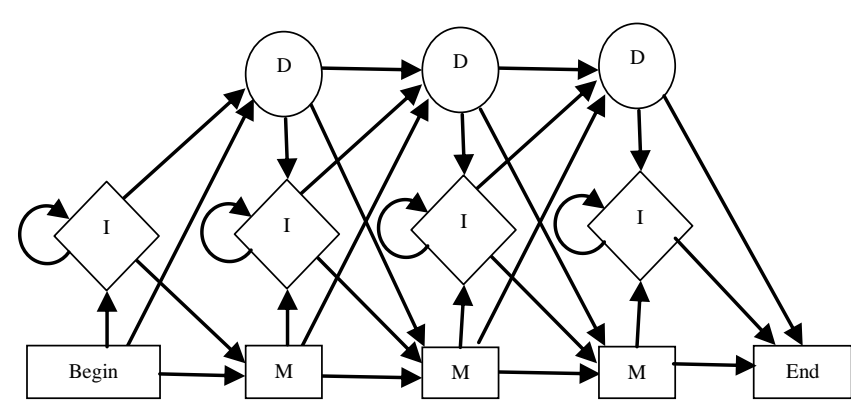

Figure 3: The Structure of a Profile HMM

The bottom lines of states are called the match (M) states, because they model the columns of the alignment. The second rows of diamond shaped states are called insert (I) states and are used to model highly variable regions in the alignment. The top lines of circular states are called delete (D) states. They do not match any residues, and they are there merely to make it possible to jump over one or more columns in the alignment, i.e. to model the situation when just a few of the sequences have a '-' in the multiple alignment at a position.

Using the profile HMM, a consensus sequence for each of the chromosomes is emitted. A consensus sequence is defined as the sequence, which is closest to all other sequences in the chromosome, and thus can be used to represent the entire set of sequences in the alignment (chromosome). An example of a consensus sequence with respect to a chromosome is depicted in the figure below:

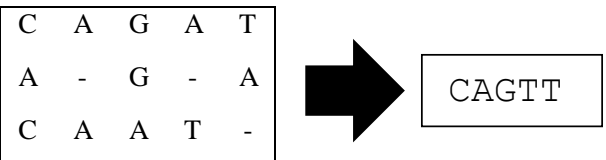

Figure 4: The Consensus Sequence 'CAGTT' obtained from a Chromosome

The Fuzzy Levenshtein Distance of each of the sequences in the chromosome is then computed from the consensus sequence.

The Levenshtein distance between two words is the minimum number of single-character edits (insertion, deletion, substitution) required to change one word into the other. Mathematically, the Levenshtein distance between two strings $\mathrm{x}, \mathrm{y}$ is given by:

$\operatorname{Lev}_{x, y}(m, n)=\left\{\begin{array}{c}\max (m, n), i f \min (m, n)=0 \\ \operatorname{Lev} v_{x, y}(m-1, n)+1 \\ \operatorname{Lev}(m, n-1)+1 \\ \operatorname{Lev}_{x, y}(m-1, n-1)+\left[x_{m} \neq y_{n}\right]\end{array}\right\}$ otherwise $\}$

The first element in the minimum corresponds to deletion (from $\mathrm{x}$ to $\mathrm{y}$ ), the second to insertion and the third to match or mismatch, depending on whether the respective symbols are the same.

The Levenshtein distance is an integer, which gives a measure of similarity between two DNA sequences. To compute the Fuzzy Levenshtein distance, the percentage similarity between two DNA sequences is computed. To transform the Levenshtein distance into a percentage, the number of edits 
required are subtracted from 1.0 and divided by the length of the longest string. The Fuzzy Levenshtein distance is obtained by multiplying the resulting value by 100 . The Fuzzy Levenshtein distance of the sequences in a chromosome from its consensus sequences is illustrated in the figure below:

\begin{tabular}{|c|c|c|}
\hline Sequence & $\begin{array}{c}\text { Consensus } \\
\text { Sequence }\end{array}$ & $\begin{array}{c}\text { Fuzzy Levenshtein } \\
\text { distance }\end{array}$ \\
\hline CAGAT & & 0.8 \\
\hline A-G-A & \multirow{2}{*}{ CAGTT } & 0.2 \\
\cline { 1 - 2 } CAAT- & & 0.6 \\
\cline { 3 - 3 } & & \\
\cline { 3 - 3 } & &
\end{tabular}

Figure 5: Fuzzy Levenshtein distances of three sequences from the consensus sequence

The fitness of a chromosome is then calculated as the average of the Fuzzy Levenshtein distances of each sequence in the alignment to the consensus alignment. For example, the fitness of the chromosome in the previous example is 0.53 .

\subsection{Genetic operators}

Selection of the chromosomes is done by the criteria of elitism, which selects the best chromosome from a population. Then the rest of the chromosomes are chosen by spinning the roulette wheel. Thus fitter individuals have a greater chance of being selected. These selected individuals are then crossed over to produce new individuals. For crossover a particular sequence of two chromosomes are interchanged. The following figure depicts an example crossover.

\begin{tabular}{|ccccc|ccccc|}
\hline C & A & G & A & T & C & A & G & A & T \\
A & - & G & - & A & - & A & G & - & A \\
C & A & A & T & - & C & A & - & A & T \\
A & - & T & A & T & - & A & T & A & T \\
C & A & G & - & T & C & A & G & - & T \\
\hline
\end{tabular}

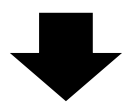

\begin{tabular}{|ccccc|ccccc|}
\hline C & A & G & A & T & C & A & G & A & T \\
A & - & G & - & A & - & A & G & - & A \\
C & A & A & T & - & C & A & - & A & T \\
- & A & T & A & T & A & - & T & A & T \\
C & A & G & - & T & C & A & G & - & T \\
\hline
\end{tabular}

Figure 6: Crossover of two chromosomes (4th sequence interchanged)

After crossover mutation is performed. In this case the position of gaps within a sequence of a chromosome has been altered, as given in the figure below:

$$
\left.\begin{array}{ccccc}
\text { C } & \text { A } & \text { G } & \text { A } & \text { T } \\
- & \text { A } & \text { G } & - & \text { A } \\
\text { C } & \text { A } & - & \text { A } & \text { T } \\
- & \text { A } & \text { T } & \text { A } & \text { T } \\
\text { C } & \text { A } & \text { G } & - & \text { T }
\end{array}\right] \rightarrow\left[\begin{array}{ccccc}
\text { C } & \text { A } & \text { G } & \text { A } & \text { T } \\
\text { A } & - & \text { G } & - & \text { A } \\
\text { C } & \text { A } & - & \text { A } & \text { T } \\
- & \text { A } & \text { T } & \text { A } & \text { T } \\
\text { C } & - & \text { A } & \text { G } & \text { T }
\end{array}\right.
$$

Figure 7: Example of mutation: gaps in sequence 2 and sequence 4 are altered
These operations are repeated over and over again for a certain number of times to produce fitter individuals.

\section{EXPERIMENTAL SETUP}

To build the profile HMMs HMMER [11] (http://hmmer.janelia.org/) has been used, a bio-sequence analysis tool using profile Hidden Markov Models. For result comparison, the T-Coffee ${ }^{[15]}$ server (http://www.tcoffee.org/), which is an online multiple sequence alignment tool, has been utilized. The experimental environment is depicted below:

$>$ Hardware

- $\quad$ Processor - Intel ${ }^{\circledR}$ Core $^{\mathrm{TM}}$ i7-3610QM CPU@ $2.30 \mathrm{GHz} \times 8$

- $\quad \mathrm{RAM}-8 \mathrm{~GB}$

- $\quad$ Disk $1000 \mathrm{~GB}$

$>$ Software

- $\quad$ Operating system - Open SUSE Kernel version 3.1.0-1.2-desktop

- $\quad$ OS type-32-bit

- Compiler used - javac

- JRE version 1.7

- HMMER 3.1

- T-Coffee Multiple Sequence Alignment Server

\section{OBSERVATIONS}

The fitness of the proposed genetic algorithm based on HMM and Fuzzy Levenshtein Distance has been compared with that of the multiple sequence alignment generated by T-Coffee. The number of generations was taken to be 100 for a population size of 100 . The graphs below depict the experimental observations.

The following graph illustrates the fitness scores of the proposed algorithm vs. T-Coffee for different sequence sizes.

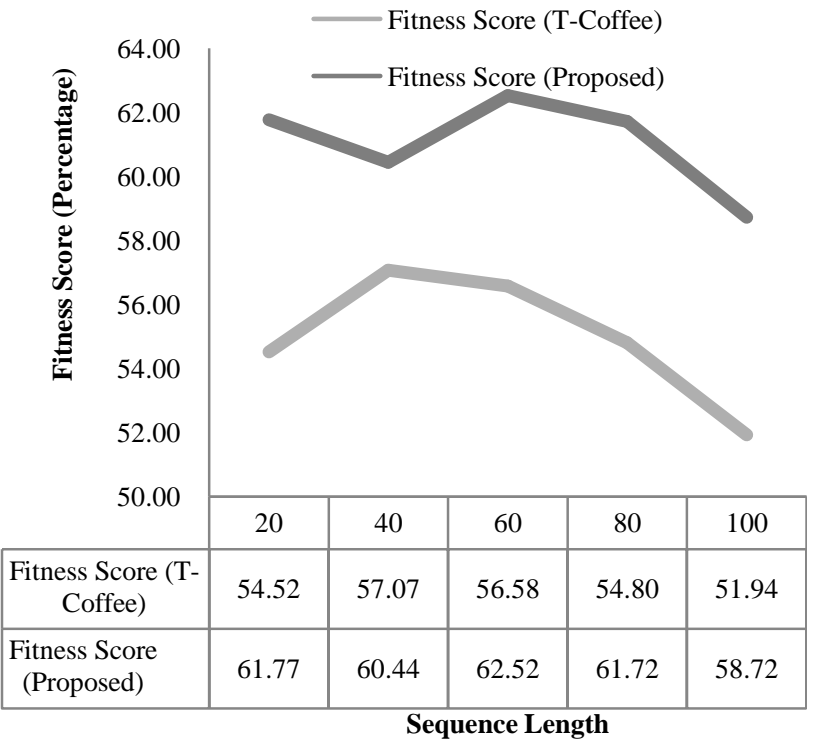

Figure 8: Sequence Length vs. Fitness Score 
The following graphs illustrate the fitness scores of the proposed algorithm vs. T-Coffee for various numbers of sequences and a fixed sequence size.

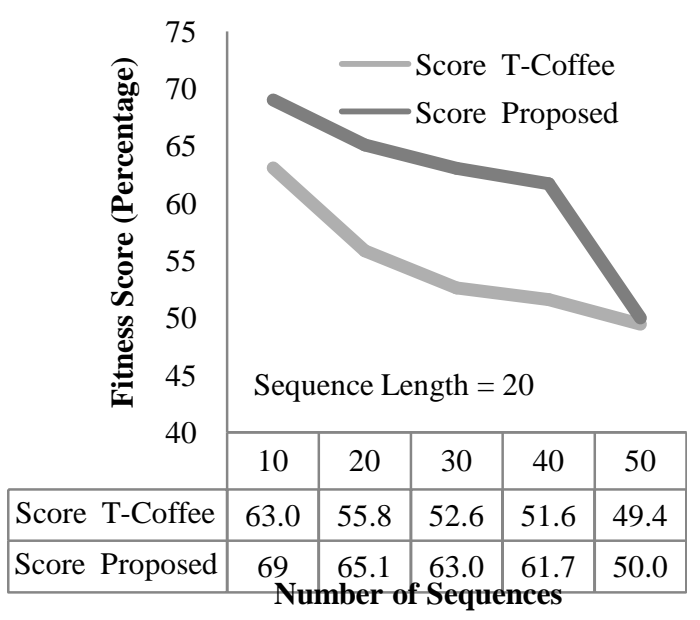

Figure 9: Number of Sequences vs. Fitness Score (Sequence Length $=20$ )

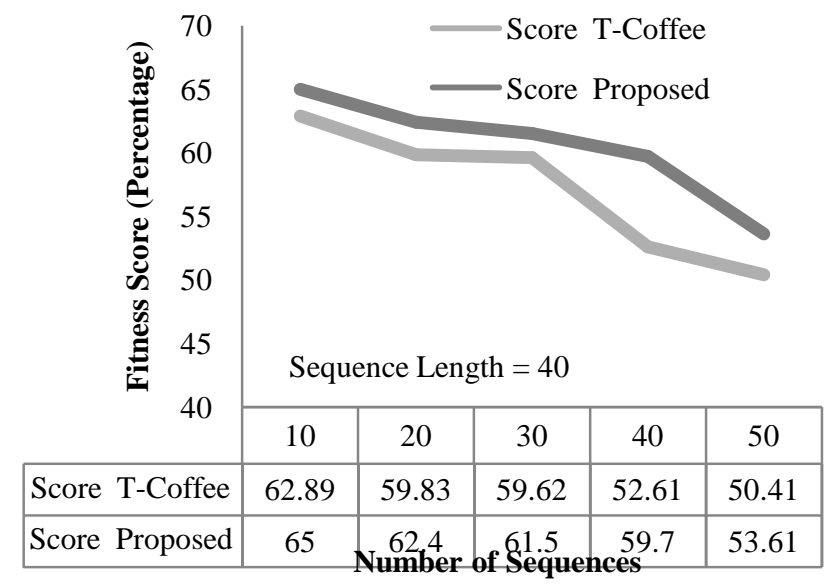

Figure 10: Number of Sequences vs. Fitness Score (Sequence Length $=40$ )

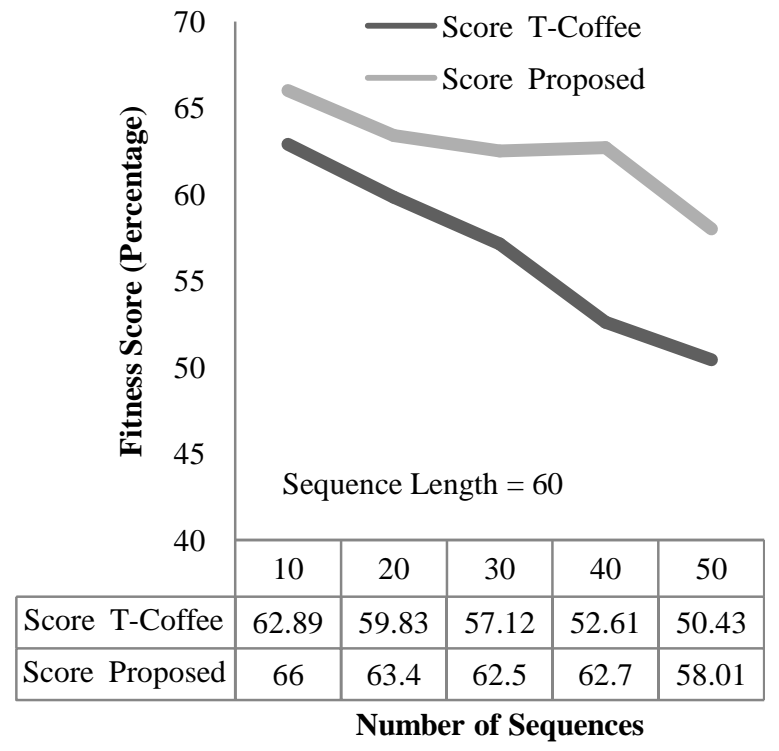

Figure 11: Number of Sequences vs. Fitness Score (Sequence Length $=60$ )

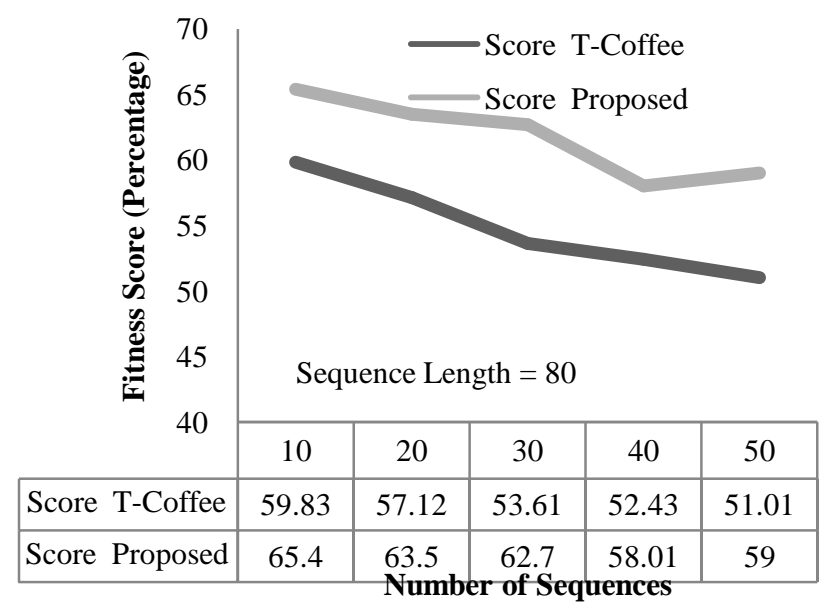

Figure 12: Number of Sequences vs. Fitness Score (Sequence Length $=80$ ) 


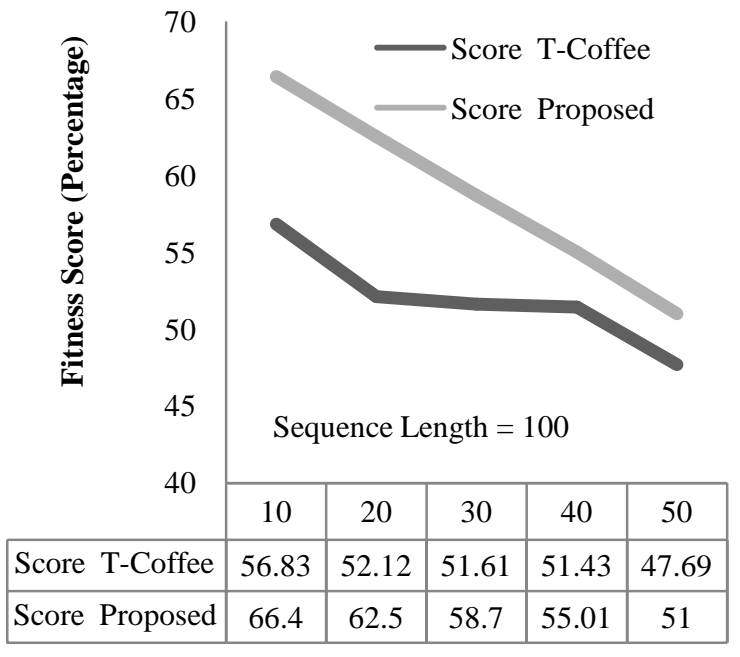

Number of Sequences

Figure 13: Number of Sequences vs. Fitness Score (Sequence Length $=100$ )

\section{CONCLUSION}

From the experimental results it can be concluded that the proposed genetic algorithm based on Hidden Markov Models and Fuzzy Levenshtein Distance works well under the given conditions and generally produces good fitness scores when compared to those of T-Coffee. It may be mentioned that the analytical study of the DNA multiple sequence alignment by genetic algorithms opens up a wide scope of investigative study with a view to explore better improvement, if any. The authors suggest the following areas of further research:

- Test for convergence with variance of population and generations.

- Improve the fitness function by incorporating affine gap costs.

- Explore better crossover and mutation mechanisms to enhance the proposed genetic evolutionary approach.

- Exploit other evolutionary computation techniques for the current problem.

\section{REFERENCES}

[1] Baum, L. and Petrie, T. (1966). Statistical inference for probabilistic functions of finite state Markov chains. The Annals of Mathematical Statistics, 37(6):1554-1563.

[2] Braun, J. V. and Muller, H.-G. (1998). Statistical methods for dna sequence segmentation. Statistical Science, 13(2):142-162.

[3] Chih-Chin Lai; Chih-Hung Wu; Cheng-Chen Ho; "Using Genetic Algorithm to Solve Multiple Sequence Alignment Problem ", International Journal of Software Engineering and Knowledge Engineering Vol. 19, No. 6 (2009)

[4] Churchill, G. (1989). Stochastic models for heterogeneous dna sequences. Bulletin of Mathematical Biology, 51:79-94. 10.1007/BF02458837.

[5] Churchill, G. (1992). Hidden markov chains and the analysis of genome structure. Computers and Chemistry, 16(2):107-115.
[6] Dong, S. and Searls, D. B. (1994) Genomics 23, 540 551.

[7] Dubin, R. E. S., Krogh, A., and Mitchison, G. (1998). Biological Sequence Analysis: probabilistic models of proteins and nucleic acids. Cambridge University Press, Cambridge.

[8] Eddy, S. R. (1995); Multiple alignment using hidden Markov models. In Rawlings, C., Clark, D., Altman, R., Hunter, L., Lengauer, T., and Wodak, S. (Eds.), Proc. of Third Int. Conf. on Intelligent Systems for Molecular Biology volume 3 pp. 114-120 Menlo Park, CA. AAAI Press.

[9] Guang-Zheng Zhang; De-Shuang Huang; "Aligning multiple protein sequence by an improved genetic algorithm," Neural Networks, 2004. Proceedings. 2004 IEEE International Joint Conference on , vol.2, no., pp. 1179- 1183 vol.2, 25-29 July 2004.

[10] Hjelmqvist, Sten (26 Mar 2012), Fast, memory efficient Levenshtein algorithm (http://www.codeproject.com/Articles/13525/Fastmemory-efficient-Levenshtein-algorithm)

[11] HMMER 3.1 (February 2013); http://hmmer.org/

[12] Hung Dinh Nguyen; Yoshihara, I.; Yamamori, K.; Yasunaga, M.; "A parallel hybrid genetic algorithm for multiple protein sequence alignment," Evolutionary Computation, 2002. CEC '02. Proceedings of the 2002 Congress on , vol.1, no., pp.309-314, 12-17 May 2002

[13] Lin, C. H., Chen, S. J., and Chen, S. M. 2003. A new method for multiple DNA sequence alignment based on genetic algorithms. Proceedings of the 2003 Joint Conference of AI, Fuzzy System, and Grey System, Taipei, Taiwan, Republic of China.

[14] Mount David W., Bioinformatics - Sequence and Genome Analysis, Cold Spring Harbor Laboratory Press, 2001.

[15] Notredame, Higgins, Heringa, T-Coffee: A novel method for multiple sequence alignments, Journal of Molecular Biology, 302 (205-217) 2000

[16] Omar, M.F.; Salam, R.A.; Rashid, N.A.; Abdullah, R.; "Multiple sequence alignment using genetic algorithm and simulated annealing," Information and Communication Technologies: From Theory to Applications, 2004. Proceedings, vol., no., pp. 455- 456, 19-23 April 2004.

[17] Ping-Teng Chang; Lung-Ting Hung; Kuo-Ping Lin; Chih-Sheng Lin; Kuo-Chen Hung; , "Protein Sequence Alignment Based on Fuzzy Arithmetic and Genetic Algorithm," Fuzzy Systems, 2006 IEEE International Conference on , vol., no., pp.1362-1367, 0-0 0.

[18] Sellers, Peter H. (1980). "The Theory and Computation of Evolutionary Distances: Pattern Recognition". Journal of Algorithms 1 (4): 359-73. doi:10.1016/01966774(80)90016-4

[19] Shyi-Ming Chen, Chung-Hui Lin, and Shi-Jay Chen, Multiple DNA Sequence Alignment Based on Genetic Algorithms and Divide-and-Conquer Techniques, International Journal of Applied Science and Engineering 2005. 3, 2: 89-100

[20] Smith-Keary. P. Molecular Genetics. Macmillan Education Ltd, London, 1991 